\title{
Drama im Aufwachraum: pädiatrisches Emergence-Delir
}

Sylvia Kramer, Martin Krebs, Claudia Spies, Shahab Ghamari, Claudia Höhne, Karin Becke,

Christoph Eich, Andreas Hoeft, Julius Wermelt, Richard K. Ellerkmann

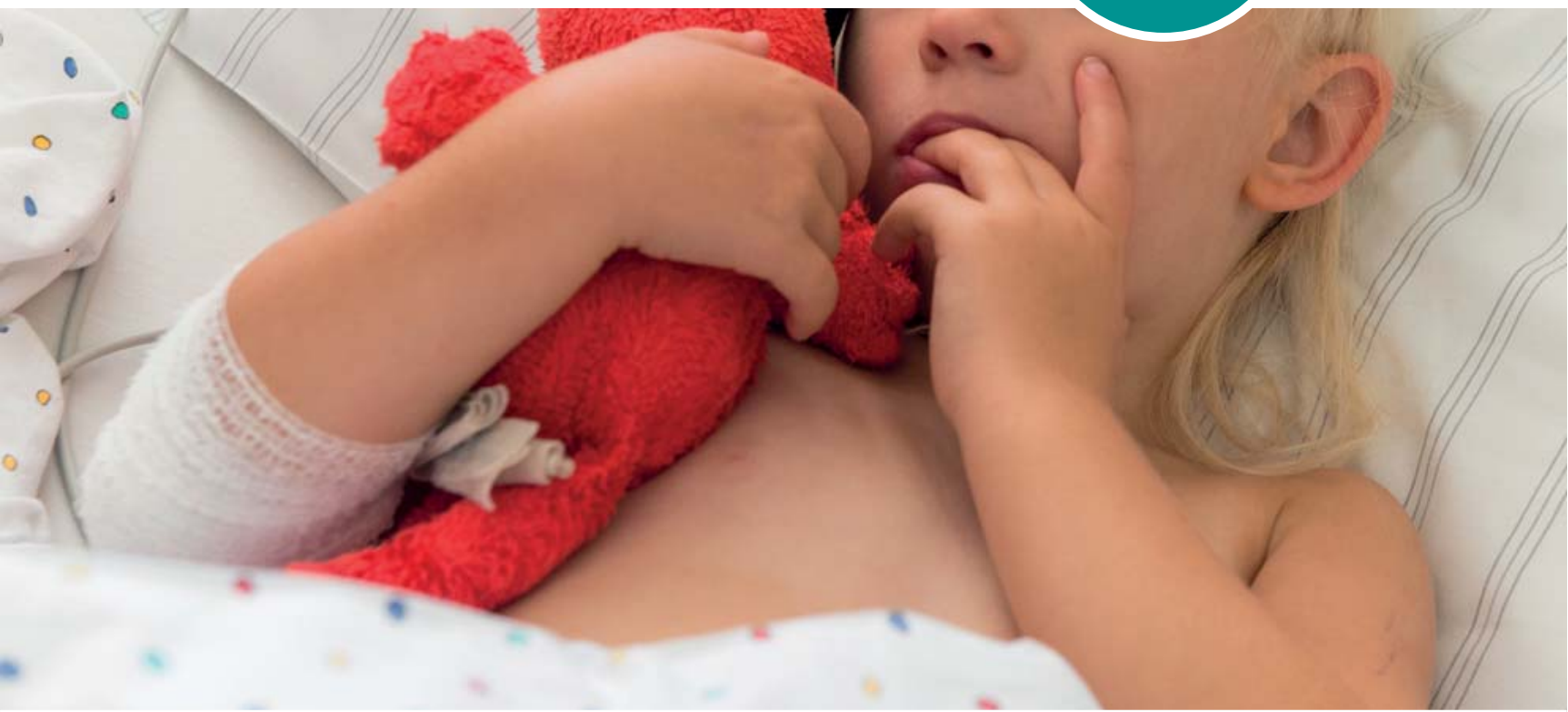

Quelle: KH Krauskopf.

Ein Bild, das Anästhesisten bekannt ist: Man kommt zurück in den Aufwachraum und das eben noch friedlich schlafende Kleinkind nach Adenotomie tobt blut- und tränenüberströmt auf dem Arm eines zutiefst beunruhigten Elternteils. Gerade als der Blick auf diese Situation fällt, zieht sich das Kind mit einer zügigen Bewegung den i.v. Zugang und stößt gleichzeitig mit dem Kopf gegen das Bett. Das Drama nimmt seinen Lauf...

\section{Einleitung}

Ein pädiatrisches Emergence-Delir (pädED) nach Sedierung oder Allgemeinanästhesie ist kein seltenes Ereignis. Beschriebene Häufigkeiten reichen bis zu $80 \%$. Das Auftreten eines pädED wird seit 1961 in der Literatur erwähnt [1]. Seit 2003 haben die tierexperimentellen Beobachtungen von Jevtovic-Todorovic eine fortlaufende Diskussion um Neurotoxizität im Säuglings- und Kindesalter angestoßen [2 -5]. Inwieweit es einen Zusammenhang zwischen Neurotoxizität in Tierexperimenten, der bislang nicht in Untersuchungen an Kindern nachgewiesen wurde, und einem pädED gibt, ist unklar.

Bekannt sind postoperative Verhaltensänderungen von Kindern nach Sedierungen/Allgemeinanästhesien mit und ohne Krankenhausaufenthalt. Diese Verhaltensände- rungen sind z. B. mit dem Post-Hospitalisation Behaviour Change Questionnaire (PHBQ) [6] abfragbar.

\section{Symptomatik des pädiatrischen Emergence-Delirs}

\footnotetext{
DEFINITION

Pädiatrisches Emergence-Delir (pädED)

Definitionsgemäß handelt es sich beim pädED um eine akute Störung von Bewusstsein und Aufmerksamkeit. Jedes Delir stellt ein Organversagen dar. Ein pädED betrifft direkt das Zielorgan jeder Sedierung oder Allgemeinanästhesie [7].
} 


$\begin{array}{ll}\text { ABKÜRZUNGEN } \\ \text { ED I/II } & \text { ED I: Delirkriterien } \\ & \text { ED II: Agitationskriterien } \\ \text { EMLA } & \text { Eutectic Mixture of local Anaesthetic } \\ \text { KUSS } & \text { kindliche Unbehagens- und Schmerzskala } \\ \text { NIRS } & \text { Near-InfraRed Spectroscopy } \\ \text { pädED } & \text { pädiatrisches Emergence-Delir } \\ \text { PAED Scale } & \text { Pediatric-Anaesthesia-Emergence- } \\ & \text { Delirium-Skala } \\ \text { PBS } & \text { Preoperative Behavior Score } \\ \text { PHBQ } & \text { Post-Hospitalisation Behaviour Change } \\ & \text { Questionnaire } \\ \text { PRIS } & \text { Propofol-Infusionssyndrom } \\ \text { TIVA } & \text { totale intravenöse Anästhesie } \\ \text { VAS } & \text { visuelle Analogskala } \\ \text { WAKKA } & \text { Wissenschaftlicher Arbeitskreis Kinder- } \\ & \text { anästhesie }\end{array}$

Um den Schweregrad des pädED zu quantifizieren, haben Sikich und Kollegen in ihrer Studie eine Skala (Pediatric Anaesthesia Emergence Delirium: PAED-Scale) von 0-20 entwickelt ( $\bullet$ Tab. 1$)$ und diese an 50 postoperativen pädiatrischen Patienten evaluiert. Für die PAED-Skala ergab sich bei einem Wert von $\geq 10$ eine Sensitivität für das Vorhandensein eines Delirs von 64\%. „Ruhelosigkeit“ und „Untröstbarkeit“ führen auf der Skala schmerzbedingt jedoch zu falsch positiven Ergebnissen.

Wie lassen sich Schmerzen von einem pädED unterscheiden?

\section{Merke}

Entscheidend für die Diagnose eines pädED sind die fehlende Kontaktierbarkeit und die fehlende Wahrnehmung der Umgebung ( $\triangleright$ Tab. 1).

Die Autoren um Locatelli haben daher in ihrer Veröffentlichung vorgeschlagen, lediglich die ersten 3 „Items“ der PAED-Skala zu berücksichtigen (=ED I). Bei Werten $\geq 9$ dieses ED-I-Scores kamen die Autoren in der Beurteilung eines postoperativen Delirs auf eine Sensitivität von 93\% und eine Spezifität von $94 \%$.
Im Sinne einer effektiven Qualitätskontrolle soll die Inzidenz des pädED im Aufwachraum anhand einer validierten Skala (beispielsweise PAED oder ED I) dokumentiert werden [7]. Schmerzen führen zu einem erheblichen Einfluss auf die Delirscores. Schmerzen müssen vor der Diagnose eines pädED ausgeschlossen werden. Deshalb muss gleichzeitig ein pädiatrischer Schmerzscore (Kindliche Unbehagens- und Schmerzskala [KUSS] oder für ältere Kinder anhand der visuellen Analogskala [VAS]) im Aufwachraum erhoben werden. Bei einem ausgeprägtem Delir findet auch bei älteren Kindern nur eine Fremdbeurteilung z.B. anhand der KUS-Skala statt. Lässt sich bei einem tobenden Kind und einem postoperativ potentiell schmerzhaften Eingriff kein Score erheben, ist die probatorische Analgetikagabe gerechtfertigt.

Wichtig für die Definition eines pädED sind die Bezugspersonen, meist die Eltern. Sie können sehr klar und spontan beurteilen „das ist nicht typisch für mein Kind“ bzw. nach einem stattgehabten pädED „das ist jetzt wieder mein Kind“. Diese Beurteilung kommt spontan und regelhaft in fast identischem Wortlaut. Bislang findet sich diese Beurteilung nicht in den Delirscores, hilft in der Praxis aber enorm bei der Differenzialdiagnose zwischen Schmerzen/Agitation und einem pädED.

Es gibt seltene weitere Differenzialdiagnosen eines pädED. Neben einer antibiotikainduzierten Psychose, die sich aufgrund der perioperativen Antibiotikaprophylaxe zeitgleich manifestieren kann [8], zählen hierzu langanhaltende Dystonien [9] sowie eine ausgeprägte retrograde Amnesie mit Verhaltensauffälligkeit nach Propofol [10].

\section{Pathophysiologie und Diagnostik}

Der zugrunde liegende Pathomechanismus des pädED bleibt unklar. Hypothesen, die sich aus klinischen Alltagsbeobachtungen ergeben, sind nicht endgültig überprüft.

Interessanterweise steigerten sich mit Einführung von Sevofluran weltweit die Beschreibungen des pädED. Inzwischen zeigte sich, dass volatile Anästhetika insgesamt das Risiko für das Auftreten eines pädED erhöhen. Insbe-

- Tab. 1 PAED-Skala aufgeteilt anhand Delir- und Agitationskriterien. Die Delirkriterien entsprechen dem ED-I-Score nach Locatelli [7]. ED: Emergence-Delir.

\begin{tabular}{|c|c|c|c|c|c|c|}
\hline & Das Kind... & gar nicht & ein wenig & etwas mehr & viel & sehr viel \\
\hline \multirow{3}{*}{$\begin{array}{l}\text { Delirkriterien } \\
\text { (ED-I-Score) }\end{array}$} & ... hält Augenkontakt zur Bezugsperson. & 4 & 3 & 2 & 1 & 0 \\
\hline & ...zeigt zielgerichtete Bewegungen. & 4 & 3 & 2 & 1 & 0 \\
\hline & ... nimmt seine Umwelt wahr. & 4 & 3 & 2 & 1 & 0 \\
\hline \multirow{2}{*}{$\begin{array}{l}\text { Agitationskrite- } \\
\text { rien (ED-II-Score) }\end{array}$} & ... ist unruhig/ruhelos. & 0 & 1 & 2 & 3 & 4 \\
\hline & ... ist untröstlich. & 0 & 1 & 2 & 3 & 4 \\
\hline
\end{tabular}




\section{Zusammenhang Blut/Gas-Verteilungskoeffizient und Emergence-Delir}

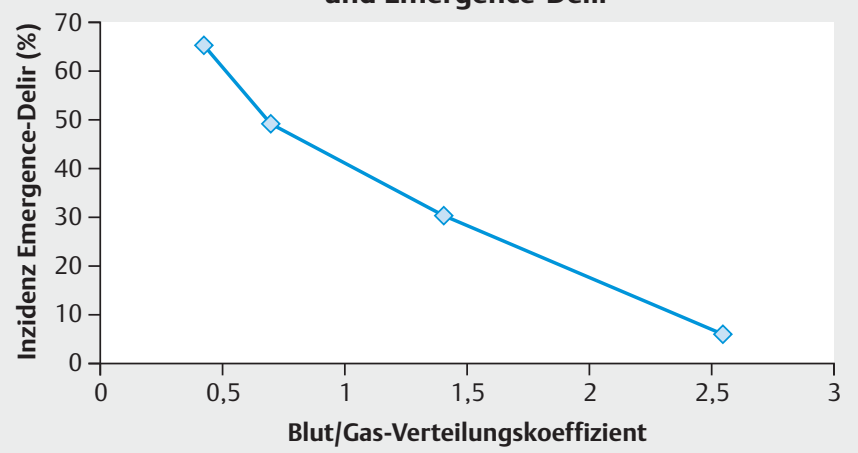

- Abb. 1 Zusammenhang zwischen dem Blut/Gas-Verteilungskoeffizient volatiler Anästhetika und der Inzidenz eines pädiatrischen EmergenceDelirs (pädED) [11]. sondere steigern schnell an- und abflutende volatile Anästhetika mit dem raschen Erwachen nach Ende der Applikation die Inzidenz eines pädED [1] ( $\mathbf{A b b} \mathbf{1}$ ).

Im Gegensatz zu den volatilen Anästhetika senkt die Anwendung von Propofol die Inzidenz eines pädED. Sowohl ein Propofol-Bolus zur Ausleitung, eine Umstellung auf eine Propofol-Kurzinfusion von $3 \mathrm{mg} / \mathrm{kgKG}$ über $3 \mathrm{~min}$ sowie eine TIVA reduzieren die Inzidenz eines pädED [1]. Eine kurze zeitlich limitierte Propofol-Anwendung im Rahmen einer TIVA nach dem Anwendungsmodell von Short erscheint für Kinder als sicher.

\section{Propofol-Infusionssyndrom}

\section{Cave}

Zu den häufigen Nebenwirkungen von Propofol während eines operativen Eingriffs bis zu 60 min gehören der reversible Anstieg sowohl der Triglyzeride und Plasmalipide als auch der bauspeicheldrüsenspezifischen Enzyme.

Bei einer Kurzzeitapplikation von Propofol über 40 min und einer Dosis von durchschnittlich $8,2 \mathrm{mg} / \mathrm{kgKG}$ traten keine relevanten Laktatspiegel bei Kindern auf [12]. Das Risiko eines PRIS bei kurzdauernden Kinderanästhesien z. B. bei HNO-Eingriffen scheint gering.

\section{PRAXIS}

Tipp

Die Gabe eines prohylaktischen Propofol-Bolus zur Ausleitung oder auch im Rahmen der Therapie eines pädED im Aufwachraum deckt sich mit der klinischen Beobachtung, einen „Reset-Button“ zu drücken und damit ein erneutes, aber ruhiges, entspanntes Aufwachen des Kindes zu ermöglichen.

\section{Elektroenzephalografische Überwachung}

Die Untersuchungen von Martin u. Mitarbeitern zur Desynchronisation des frontalen EEG könnten dazu kongruent sein. Sie zeigten, dass ein pädED mit Erwachen aus einem tiefen unbestimmten EEG-Stadium vor dem Einsetzen eines schlafähnlichen EEG einhergeht. Dies deckt sich mit der klinischen Beobachtung von erneutem Propofol-induziertem Schlaf mit ruhigem Erwachen im Gegensatz zum plötzlichen abrupten Erwachen ins pädED hinein.

In der Untersuchung von Martin et al. wurde direkt nach dem Ende der Sevofluran-Zufuhr bei späterem pädED eine erhöhte Aktivität im Frontallappen beobachtet [1]. Die EEG-Überwachung jeder Kindernarkose ist noch nicht klinische Routine. In der Klinik der Autoren erfolgt eine einfache Überwachung des Roh-EEG jenseits kalkulierter Indizes. Die Überwachung ist auch für Säuglinge nutzbar und nach Training einfach nutzbar.

Allein die Option einer Überwachung des Zielorgans Gehirn steigert die Aufmerksamkeit des Anästhesisten für die kritischen Bereiche Hirnaktivität/pädED/Neurotoxizität. Die verwendeten Medikamente werden während der Narkoseführung in der Dosis re-evaluiert und entsprechend angepasst. Dies führt zu einer verstärkten ständigen kritischen Kontrolle der Narkoseführung und Aufrechterhaltung der kindlichen Homöostase.

\section{Nahinfrarot-Spektroskopie}

Die Nahinfrarot-Spektroskopie (NIRS) als weiteres nichtinvasives Monitoring unterstützt insbesondere im Bereich der Kinderkardioanästhesie und der anästhesiologischen Versorgung von Kindern mit angeborenen Herzfehlern das anästhesiologische Management und misst laut Herstellerangaben die hirnvenöse Sauerstoffsättigung. NIRSVeränderungen werden u.a. bei Änderungen des Blut-

\section{FALLBEISPIEL}

Fortsetzung: Deeskalation und erneute Sedierung

Die zutiefst beunruhigten Eltern des eingangs beschriebenen tobenden Kleinkinds machen sich Vorwürfe, der präoperativen oralen Gabe von Midazolam zugestimmt und nicht auf ihre Anwesenheit bei der Einleitung bestanden zu haben. Um schnell zu deeskalieren und dem Kind weiteren Stress zu ersparen, unterstützen die Eltern die nasale Sedierung. Nach nasaler Applikation von Clonidin und Esketamin wird das Kind in stabiler Seitenlage mit re-etabliertem Monitoring für einen ruhigeren Aufwachversuch zurück ins Bett gelegt. Für eine eventuelle erneute Anlage eines i. v. Zugangs werden an den Füßen EMLA-Pflaster geklebt und unter den Socken versteckt. 
drucks und damit der zerebralen Homöostase beobachtet. NIRS in Zusammenhang mit pädED wurde bislang noch nicht in randomisierten kontrollierten Studien untersucht.

\section{Prävention}

Spannend bleibt die Frage nach der Identifikation gefährdeter Kinder. Die Identifikation von Risikofaktoren könnte hilfreich sein, um regelhaft eine Risikoreduktion zu erzielen.

\section{Risikofaktoren, Risikoreduktion}

\section{Angst}

Präoperative Angst ist als Risikofaktor für ein pädED beschrieben. Es gibt den Versuch eine Risikoabschätzung für ein pädED anhand von präoperativer Angst und evtl weiteren Risikofaktoren zu entwickeln $[13,14]$. Die von Hino und Mitarbeitern [14] entwickelte Risikoskala ( $\triangleright$ Tab. 2) ermöglicht mit $90 \%$ Sensitivität eine Vorhersage zum pädED bei etwa $60 \%$ der untersuchten Patienten. Dies bietet eine Möglichkeit der Risikoabschätzung, die bislang nicht im Klinikalltag etabliert ist.

\section{Alter}

Kleinkinder und Vorschulkinder sind am häufigsten von einem pädED betroffen. Im Gegensatz zu Erwachsenen und Schulkindern ist es Kindern in diesem Alter im Rahmen ihrer emotionalen Entwicklung nicht möglich, Stress (bedingt durch Nüchternheit, fremde Umgebung, Trennung von den Eltern und anderen äußeren Einflüssen) zu kompensieren. Die niedrigere Frustrationstoleranz in diesem Alter führt eventuell zu der erhöhten Inzidenz eines pädED bei Kindern im Vorschulalter [7]. Eltern kennen diese Beobachtung aus dem Alltag: Fehlt das Lieblingskuscheltier zum richtigen Zeitpunkt, kann sich schnell ein ausgeprägtes Drama entwickeln! Perioperativ fehlende Eltern/Bezugspersonen können schnell zu ausgeprägten Angstreaktionen führen.

\section{ADVANCE}

Zusammen mit der Risikoabschätzung stellt sich die Frage nach der Prävention eines pädED. Im Rahmen des ADVANCE-Studienprotokolls wurde die gesamte Familie intensiv auf den OP-Tag vorbereitet und die Eltern gezielt in unterstützender Kommunikation geschult (s. Infobox). Mit ADVANCE zeigte sich eine deutliche Reduktion des pädED.
- Tab. 2 Risikoscore für die Entwicklung eines pädiatrischen EmergenceDelirs [14].

\begin{tabular}{|c|c|c|}
\hline Parameter & & Score-Wert \\
\hline Alter & & 9 minus Alter \\
\hline \multirow[t]{3}{*}{ Art des Eingriffs } & Strabismus-OP & 7 \\
\hline & Tonsillektomie & 7 \\
\hline & andere OP & 0 \\
\hline \multirow[t]{3}{*}{ PBS } & schreiend & 4 \\
\hline & $\begin{array}{l}\text { weinerlich/ängstlich, } \\
\text { dabei aber zugänglich }\end{array}$ & 2 \\
\hline & ruhig & 0 \\
\hline \multirow[t]{4}{*}{ Anästhesiedauer } & $>2 \mathrm{~h}$ & 4 \\
\hline & $1-2 \mathrm{~h}$ & 2 \\
\hline & $<1 \mathrm{~h}$ & 0 \\
\hline & Total & ED Risikoscore: ... \\
\hline Auswertung & $\begin{array}{l}\text { ES Risikoscore > 11: } \\
\text { pädED sehr wahr- } \\
\text { scheinlich }\end{array}$ & \\
\hline
\end{tabular}

PBS: Preoperative Behavior Score

Außerhalb von Studienprotokollen scheint das Vorgehen aufgrund fehlender Ressourcen nicht durchführbar. Jegliche intensive Beschäftigung mit dem Kind (Singen/Musik, Clown-Doktoren, I-Phones/Tablets, 3-D-Videos) und damit verbunden empathische menschliche Anteilnahme und Unterstützung in einer neuen Situation reduziert die Angst. Reduzierte präoperative Angst geht in allen Untersuchungen mit einer reduzierten Inzidenz eines pädED einher [1].

Für Elternanwesenheit bei der Einleitung gilt genau wie im ADVANCE-Protokoll: Angstreduktion hilft, die Inzidenz eines pädED zuverringern. Deshalb sollten das Kind und seine Familie intensiv auf den OP-Tag vorbereitet werden. 
Merke

Durch gute Vorbereitung von Kind und Familie kann die Angst reduziert und die Inzidenz eines pädED verringert werden.

Vielfach wünschen sich Eltern, ihre Kinder bis in die Einleitungsphase zu begleiten. Der Vorteil für die Kinder hängt stark von der Vorbereitung der Eltern ab.

\section{Propofol}

PRAXIS

Tipp

Eine praxistaugliche Variante stellt z. B. bei gut geklebten EMLA-Pflastern und problemloser Anlage eines i.v. Zugangs die Propofolsedierung in Anwesenheit der Eltern oder die nasale Sedierung mit Ketanest Esketamin/Clonidin dar [1].

\section{Midazolam}

Auch durch Midazolam medikamentös reduzierte Angst senkt die Inzidenz eines pädED. Gleichzeitig stehen Benzodiazepine im Verdacht, potenziell delirogen zu sein. Dies spiegelt sich in den Untersuchungsergebnissen wider. Immer, wenn mit Midazolam in ausreichender Dosierung die präoperative Angst der Kinder gemindert wurde, sinkt die Inzidenz eines pädED. Ist der Eingriff aber sehr kurz, d. h. kürzer als die Wirkdauer des Midazolams, steht nicht die Angstreduktion, sondern die Induktion eines pädED im Vordergrund. Dementsprechend gibt es Fallberichte zu einer erfolgreichen pädED-Therapie durch den Antagonisten Flumazenil [7].

\section{Cave}

Die nasale Gabe von Midazolam brennt! Bei einem pädED steigert dies eventuell das Drama im Aufwachraum! Sollte das Kind während der nasalen Applikation abrupt den Kopf wegdrehen, kann zusätzliches Nasenbluten auftreten.

\section{Familiäre Besonderheiten beim Abbaumuster}

Genetische Polymorphismen und ihr Einfluss auf im anästhesiologischen Alltag häufig verwendete Medikamente finden bislang wenig Beachtung. Diese betreffen vielfach auch das CYP3A4-Isoenzym mit Folgen für die Verstoffwechslung verschiedenster Anästhetika und Analgetika. Für das pädED wichtig sind u.a. Midazolam respektive der Antagonist Flumazenil. Bei Erhebung der anästhesiologischen Familienanamnese, wie es sich im Speziellen für eine Kinderanästhesie und die erste Allgemeinanästhesie im Leben empfiehlt, können wegweisende Befunde erhoben werden: „Nach drei Tagen ist die Narkose erst wieder abgeklungen“, „bei mir wirkt Dormicum nicht“ u. Ä. [15]. Diese unterschiedlichen Abbaumuster können die erfolgreiche Anwendung bei Flumazenil beim pädED miterklären.

\section{$\alpha_{2}$-Agonisten}

Ein anderer erfolgreicher Weg der Prophylaxe besteht in der Anwendung von $\alpha_{2}$-Agonisten. Clonidin sowie als weiterer Vertreter der $\alpha_{2}$-Agonisten Dexmedetomidin führen unabhängig vom Applikationsweg zu einer signifikanten Reduktion des pädED [1]. Ob Dexmedetomidin auch zu einer Reduktion von postoperativen negativen Verhaltensänderungen (PHBC), wie z. B. Albträumen nach Krankenhausaufenthalt, führt, ist Gegenstand einer anlaufenden Untersuchung in Australien [6].

\section{Zerebrale Homöostase}

Eine weitere Hypothese zur Pathophysiologie des pädED bezieht sich auf Veränderungen der zerebralen kindlichen Homöostase. Diese Veränderungen können z. B. im Rahmen von Infektionen/Sepsis, Blutdruckschwankungen oder einer katabolen Stoffwechsellage auftreten. Das kindliche Delir auf Intensivstationen hat einen Bezug zu Infektionen. Für das pädED gibt es den Nachweis dieses Zusammenhangs bislang nicht. Das pädED wird eher bei jungen gesunden Kindern beschrieben. Gleichzeitig sind Kinder für HNO-Eingriffe eine Risikogruppe für das pädED [7]. Diese Kinder leiden vielfach unter Dauerinfekten.

Veränderungen der zerebralen Homöostase treten auch im Rahmen langer Nüchternzeiten auf $[16,17]$. Diese katabole Stoffwechsellage könnte ein pädED triggern. Richtungsweisende Untersuchungsergebnisse stehen aus. Manche pädiatrische anästhesiologische Zentren haben ihre Nüchternzeiten („trinken bis Abruf in den OP“) für Kinder geändert [18]. Sollte die Inzidenz des pädED an diesen Zentren sinken, wäre das ein weiterer Anhaltspunkt, um den Einfluss der zerebralen Homöostase auf die Entwicklung eines pädED zu prüfen.

\section{PRAXIS}

Tipp

Auf jeden Fall sollten die Nüchternzeiten für das Trin-

ken klarer Flüssigkeit vor dem Anästhesie-/Sedierungsbeginn kurz gehalten und organisatorisch auf kurze Nüchternzeiten geachtet werden [16].

Eine Aufklärung der Eltern/Bezugspersonen über die Möglichkeit eines pädED erfolgt oft nicht im Rahmen des Anästhesieaufklärungsgesprächs. Bei Kindern mit erhöhtem Risiko ist es nach Ansicht der Verfasser notwendig, dass Eltern informiert und über mögliche Therapiemaßnahmen aufgeklärt werden. Grundsätzlich sollten die Eltern über die Möglichkeit des Auftretens postoperativer Verhaltensveränderungen informiert und gleichzeitig darauf hingewiesen werden, dass diese grundsätzlich keine bekannten negativen Folgen haben. 
PRAXIS

Tipp

Die Eltern sollten Geschwisterkinder ggf. auf post-

operative Verhaltensänderungen des Patienten vor-

bereiten.

\section{Therapeutisches Vorgehen}

Für die Therapie des pädED sollten zuerst immer die in der Übersicht genannten Basismaßnahmen durchgeführt werden. Danach erfolgen dann die rasche medikamentöse Therapie zur Stressreduktion des Kindes und die aufklärende Beruhigung der gesamten Familie.

\section{THERAPIE}

Basismaßnahmen zur Behandlung eines pädiatrischen Emergence-Delirs

- gute Analgesie

- Ruhige Aufwachumgebung und Anwesenheit der Eltern

- falls möglich Getränke und/oder kleine Schnacks anbieten

- vor greller Sonne/Licht abschirmen

- Überhitzung vermeiden

Das klinisch angenommene pädED sollte mit einer Skala quantifiziert werden [1]. Hierzu kann z. B. die PAED-Skala in Zusammenhang mit der KUSS-Skala dienen, um Schmerzen als Ursache für das pädED abschätzen zu können. Bei einem deliranten Kind findet nur eine Fremdbeurteilung Anwendung. Kann eine schmerzbedingte Ursache nicht ausgeschlossen werden, können nach Ausschöpfung der nichtmedikamentösen Maßnahmen unter pulsoxymetrischer Kontrolle i.v. Opioide zum Einsatz kommen.

Wenn eine Fremd- oder Eigengefährdung besteht, hat sich der Einsatz von i. v. Anästhetika bewährt. Hierbei kann die Gabe von Propofol in einer Dosierung von 0,5-1 mg/ kgKG unter pulsoxymetrischer Kontrolle und Beatmungsbereitschaft Anwendung finden. Alternativ kann auch die Gabe von Clonidin in einer Dosierung von $2 \mu \mathrm{g} / \mathrm{kgKG}$ oder die Gabe von 0,5-1 mg/kg/KG Esketamin erfolgen.

Der Wissenschaftliche Arbeitskreis Kinderanästhesie (WAKKA) eruierte in einer deutschlandweiten Umfrage unter Kinderanästhesisten die präferierte Therapieoption. Die höchste Zufriedenheit unter den Kinderanästhesisten hinsichtlich der Wirkung einer medikamentösen Therapie wurde Propofol zugeordnet ( $\bullet$ Abb. 3) [7].

Interessanterweise konnten Almenrader und Kollegen unterschiedliche Herangehensweise zu Prophylaxe und

\section{FALLBEISPIEL}

\section{Fortsetzung: Gespräch mit Eltern}

Nachdem im Aufwachraum jetzt wieder Ruhe herrscht, wird mit den Eltern ausführlich gesprochen. Für die Eltern ist wichtig zu wissen, ob diese Episode negative Folgen für ihr Kind haben könnte. Außerdem bedeutsam: Wird sich das Kind an diese Situation erinnern? Und weiß man, ob das Delir bei der nächsten Narkose wieder auftreten wird? Nach dem aktuellen Wissensstand sind direkte negative Folgen unwahrscheinlich. Nach einer Krankenhausbehandlung kann es immer mal wieder Kinder mit Problemen, wie z. B. erneutes Bettnässen, gesteigerter Aggressivität oder Angst, geben. Größere Kinder berichten eventuell spontan vom pädED.

Wichtig für die Eltern ist, dass sie den Erzählungen der Kinder, ihren Albträumen unbedingt zuhören sollten. Für das Kind war der Albtraum real und oft angstbesetzt. Unbedingt sollte man eine stattgehabte pädED-Episode für die nächste Narkose mitangeben. Mit dieser Information können alle bekannten evidenzbasierten Prophylaxestrategien ergriffen werden.

Jetzt sind die drängendsten Fragen der Eltern geklärt. Bieten Sie im Gespräch Getränke an und ermöglichen Sie den Eltern ggf. abwechselnd eine Pause außerhalb des Aufwachraums. Eltern sind nach einem pädED des Kindes meist erschöpft und dankbar für Unterstützung.

Therapie des pädED innerhalb Europas zeigen ( $\bullet$ Abb. 4 und 5). Verglichen wurden Prophylaxe und Therapieregime zwischen Großbritannien und Italien. Anders als in Deutschland bevorzugten die italienischen Kollegen Midazolam zur Prophylaxe und Therapie. In Großbritannien fanden weniger Medikamente als vielfach Eltern bzw. der Faktor Zeit bei üblicherweise 1:1-Betreuung im Kinderaufwachraum Anwendung [19].

Den Anwendungsbeobachtungen zur Therapie des pädED folgten bislang keine randomisiert-kontrollierten Studien. Eine Studie untersuchte den therapeutischen Effekt von Physostigmin im Vergleich zu Placebo, ohne einen klaren Vorteil für Physostigmin erkennen zu können [7].

Ein langanhaltendes Delir mit wiederholten Episoden trotz medikamentöser Therapie spricht eventuell auf Antipsychotika an. Risperidon als Antagonist am $5 \mathrm{HT}_{3^{-}}$, D2-, $\alpha_{1}$ - und $\alpha_{2}$-Rezeptor und Histaminantagonist erwies sich im Fallbericht als erfolgreiche antipsychotische Rescue-Therapie eines langanhaltenden pädED [20].

\section{FAZIT}

Der zugrunde liegende Pathomechanismus des pädED ist weiterhin unklar. Hypothesen, die sich aus klinischen Alltagsbeobachtungen ergeben, sind nicht endgültig überprüft. 


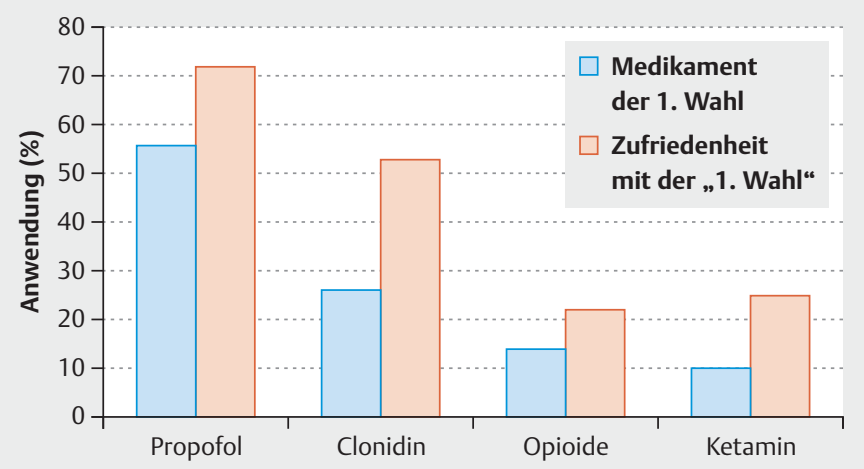

- Abb. 2 Ergebnisse der Umfrage zur Therapie des pädiatrischen Emergence-Delirs in Deutschland [7].

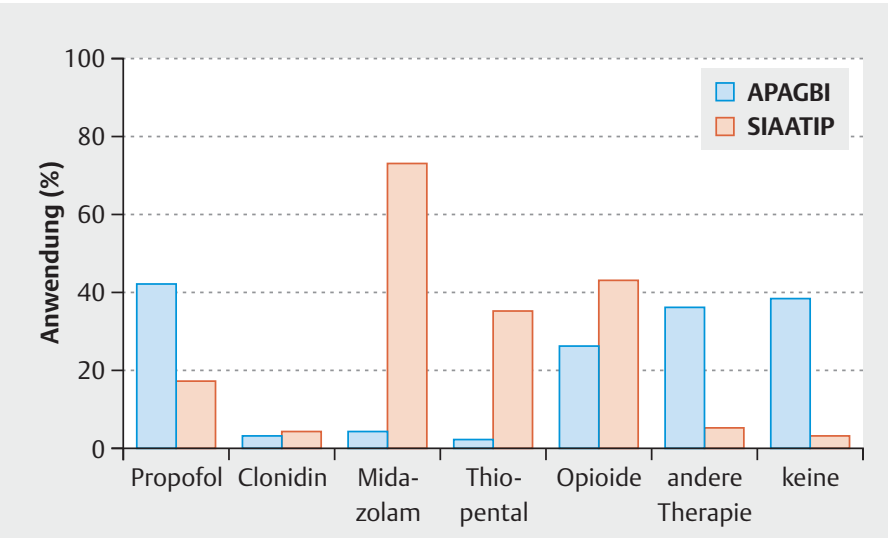

- Abb. 3 Prophylaxe des pädiatrischen Emergence-Delirs im europäischen Vergleich [19]. APAGBI: Association of Paediatric Anaesthetists of Great Britain and Ireland; SIAATIP: Italian Paediatric Society of Anaesthesia, Analgesia and Intensive Care.

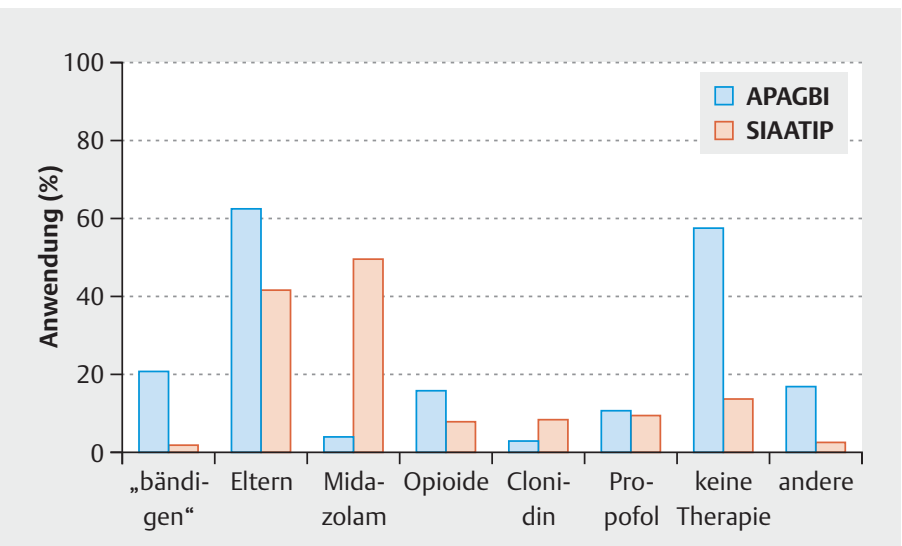

- Abb. 4 Therapie des pädiatrischen Emergence-Delir (pädED) im europäischen Vergleich [19].

\section{Rezidivprophylaxe}

\section{Merke}

Kinder und deren Familien, welche die Erfahrung eines pädED gemacht haben, berichten bei erneut notwendiger Narkose nicht unbedingt spontan von dieser Erfahrung. Es sollte daher gezielt danach gefragt werden.

Ältere Kinder können, falls sie sich erinnern, von einem früher durchgemachten pädED berichten. Diese Berichte ähneln sich in Bezug auf „Angst/Angst zu sterben/Angst zu ersticken“, oder „Wut“. Die Kinder beschreiben dies eventuell als Albtraum. Insgesamt ist es eine Erinnerung an eine stark negative, emotionale Situation.

Kinder und Familien haben die Erfahrung eventuell einfach als Teil der perioperativen Prozedur abgespeichert und gehen davon aus, dass es genauso wieder geschehen wird. Es gibt aktuell keine Forschungsergebnisse zur Rezidivhäufigkeit eines pädED. Vielfach sind die ehemals kleinen Kinder, die das pädED erlitten, bis zur nächsten notwendigen anästhesiologischen Versorgung älter geworden. Ein Teil der Kinder hat aber nur einen kurzen Abstand zum pädED, u.a. bei Metallentfernung nach Frakturstabilisierung, sequenzielle Narkosen aufgrund einer Leukämie mit Diagnostik, Probenentnahmen oder der Anlage eines zentralvenösen Katheters.

Es empfiehlt sich, eine Rezidivprophylaxe im Rahmen der Möglichkeiten anzustreben. Dabei zählt jede mögliche Stellschraube:

- Kann die präoperative Angst weiter gemindert werden?

- Ist eine Anpassung des Anästhesieregimes, z. B. über zusätzliche Regionalanästhesie oder Zusatz von $\alpha_{2^{-}}$ Agonisten, möglich?

- Ist die Analgesie postoperativ verbesserbar?

- Können die Kinder ihr Lieblingskuscheltier oder Spielzeug mitbringen?

- Dürfen Eltern beruhigende Musik - natürlich mit Kopfhörern - mitbringen?

Nach einem ausgeprägtem pädED gibt es außer auf dem Anästhesieprotokoll nur die Möglichkeit der Dokumentation im Entlassungsbrief des Kindes. Dieser Entlassungsbrief kann gemeinsam mit den chirurgischen Kollegen geschrieben werden oder als separater Entlassungsbrief der anästhesiologischen Klinik erstellt werden. Wichtig für die Rezidivprophylaxe des pädED ist die Erfassung des präoperativen Status, der intraoperative Verlauf inklusive Volumenstatus und die postoperative Behandlung des pädED im Aufwachraum.

Forschung zur Entwicklung eines pädED im normalstationären Bereich jenseits des anästhesiologisch betreuten Aufwachraums und der Intensivstation [21] fehlen. Anek- 
dotische Fallberichte zum pädED nach pädiatrischen Sedierungen existieren.

\section{KERNAUSSAGEN}

- Das pädiatrische Emergence-Delir (pädED) rückt aufgrund der Debatte um Neurotoxizität von Anästhetika bei kleinen Kindern erneut in den Fokus. Die 2017 von der Europäischen Gesellschaft für Anästhesiologie publizierte Leitlinie zu Prävention und Therapie kann eine sinnvolle Unterstützung der klinischen Tätigkeit sein.

- Insbesondere die zügige und konsequente Behandlung von Schmerzen bei kleinen Kindern und die konsequente Diagnose eines pädED mittels validierter Skalen ermöglicht es - dank der Verbreitung von Patient-Data-Management-Systemen -, in Zukunft eine reelle Inzidenz des pädEDs anzugeben.

- In der Prävention des pädED liegt der Schwerpunkt auf der Reduktion der präoperativen Angst der Kinder, egal, ob dies durch ein auf das Kind fokussiertes Kinderanästhesieteam zusammen mit den Eltern, Musik, Clowns, Smartphones/Tablets oder eine medikamentöse Prämedikation erzielt wird.

- Medikamentöse pädED-Prophylaxe durch perioperative Anwendung von $\alpha_{2}$-Agonisten und die Verwendung von Propofol als Ausleitungsbolus oder TIVA erscheint gleichzeitig sinnvoll.

- Postoperativ ermöglicht eine ruhige Aufwachumgebung ein entspanntes delirfreies Aufwachen.

- Postanästhesiologische Visiten mit strukturiertem Erfassen von Veränderungen des kindlichen Verhaltens respektive schriftliche Fragebögen werden in Zukunft Auskunft über das pädED auf den Normalstationen geben. Durch strukturierte Nachbefragungen im weiteren Verlauf werden auch postoperative unerwünschte Verhaltensänderungen und deren möglicher Zusammenhang mit dem pädED erfasst werden können.

- Die 2017 von der Europäischen Gesellschaft für Anästhesiologie (ESA) publizierte Leitlinie zu Prävention und Therapie kann eine sinnvolle Unterstützung der klinischen Tätigkeit sein.

\section{Interessenkonflikt}

Die Autoren geben an, dass keine Interessenkonflikte vorliegen.

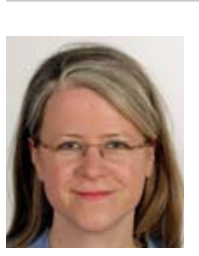

\section{Sylvia Kramer}

Dr. med., DESA. 2011 Trainee der European Society for Anesthesiology: Kinderkardioanästhesiologie. 2010 Fachärztin für Anästhesiologie. 2011 Zusatzbezeichnung Notfallmedizin. 2014 Zusatzbezeichnung Spezielle Intensivmedizin. Seit 2013 Oberärztin im Bereich Kinderklinik, Campus Virchow-Klinikum, Charité - Universitätsmedizin Berlin.

\section{Claudia Spies}

Univ.-Prof. Dr. med. Seit 2005 Direktorin der Klinik für Anästhesiologie mit Schwerpunkt operative Intensivmedizin, Campus Charité Mitte und Campus Virchow-Klinikum, Charité Universitätsmedizin Berlin.

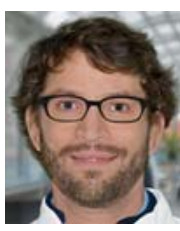

\section{Martin Krebs}

Dr. med. 1998-2005 Studium der Humanmedizin an der Universität Ulm. 2012 Facharzt für Anästhesiologie, Zusatzbezeichnungen Notfallmedizin, Hämostaseologie. Seit 2016 Oberarzt Kinderanästhesie der Klinik für Anästhesiologie mit Schwerpunkt operative Intensivmedizin der Charité - Universitätsmedizin Berlin am Campus Virchow-Klinikum. Wissenschaftliche Schwerpunkte: Kinderanästhesie, Hämostaseologie.

\section{Shahab Ghamari}

Dr. med. Facharzt an der Klinik und Poliklinik für Anästhesiologie und Operative Intensivmedizin am Universitätsklinikum Bonn.

\section{Claudia Höhne}

Prof. Dr. med. Chefärztin, Klinik für Anästhesiologie, Intensivmedizin und Schmerztherapie, DRK Kliniken Berlin-Köpenick. Schriftführerin des Wissenschaftlichen Arbeitskreises Kinderanästhesie (WAKKA) der DGAI.

\section{Karin Becke}

Dr. med. Chefärztin der Abteilung für Anästhesie und Intensivmedizin der Cnopf'schen Kinderklinik/Klinik Hallerwiese in Nürnberg. 1. Sprecherin des Wissenschaftlichen Arbeitskreises Kinderanästhesie (WAKKA) der DGAI und Vorsitzende des Wissenschaftlichen Subkomitees Kinderanästhesie der Europäischen Gesellschaft für Anästhesiologie (European Society of Anaesthesiology, ESA).

\section{Christoph Eich}

Prof. Dr. med. Chefarzt der Abteilung Anästhesie, Kinderintensiv- und Notfallmedizin im Kinder- und Jugendkrankenhaus Auf der Bult in Hannover. 2. Sprecher des Wissenschaftlichen Arbeitskreises (WAKKA) Kinderanästhesie der DGAI.

\section{Andreas Hoeft}

Prof. Dr. med. Direktor der Klinik und Poliklinik für Anästhesiologie und Operative Intensivmedizin am Universitätsklinikum Bonn. 


\section{Julius Wermelt}

Dr. med. Oberarzt der Klinik für Anästhesiologie am Klinikum der Ludwig-Maximilians-Universität München.

\section{Richard K. Ellerkmann}

Prof. Dr. med., DESA. Direktor der Klinik für Anästhesiologie und operative Intensivmedizin, Klinikum Dortmund. Wissenschaftlicher Mitarbeiter der medizinischen Fakultät Bonn.

\section{Korrespondenzadresse}

\section{Dr. med. Sylvia Kramer, DESA}

Klinik für Anästhesiologie m. S. operative Intensivmedizin Charité - Universitätsmedizin Berlin

Campus Charité Mitte und Campus Virchow-Klinikum

Augustenburger Platz 1, 13353 Berlin

Sylvia.Kramer@charite.de

\section{Wissenschaftlich verantwortlich} gemäß Zertifizierungsbestimmungen

Wissenschaftlich verantwortlich gemäß Zertifizierungsbestimmungen für diesen Beitrag ist Dr. med. Sylvia Kramer, Berlin.

\section{Literatur}

[1] Aldecoa C, Bettelli G, Bilotta F et al. European Society of Anaesthesiology evidence-based and consensus-based guideline on postoperative delirium. Eur J Anaesthesiol 2017; 34: 192-214. doi:10.1097/EJA.0000000000000594

[2] Block RI, Thomas J], Bayman EO et al. Are anesthesia and surgery during infancy associated with altered academic performance during childhood? Anesthesiology 2012; 117: 494503. doi:10.1097/ALN.0b013e3182644684

[3] Davidson AJ, Disma N, de Graaff JC et al.; GAS consortium. Neurodevelopmental outcome at 2 years of age after general anaesthesia and awake-regional anaesthesia in infancy (GAS): an international multicentre, randomised controlled trial. Lancet 2016; 387: 239-250. doi:10.1016/S0140-6736(15)00608$\mathrm{X}$

[4] Hu D, Flick RP, Zaccariello MJ et al. Association between exposure of young children to procedures requiring general anesthesia and learning and behavioral outcome in a populationbased birth cohort. Anesthesiology 2017; 127: 227-240. doi:10.1097/ALN.0000000000001735

[5] Ing CH, DiMaggio C], Whitehouse AJ et al. Neurodevelopmental outcomes after initial childhood anesthetic exposure between ages 3 and 10 years. J Neurosurg Anesthesiol 2014; 26: 377-386. doi:10.1097/ANA.0000000000000121

[6] Lee-Archer P, McBride C, Paterson R et al. Does dexmedetomidine given as a premedication or intraoperatively reduce posthospitalisation behavior change in children? A study protocol for a randomised controlled trial in a tertiary paediatric hospital. BMJ Open 2018; 8: e019915. doi:10.1136/bmjopen-2017019915

[7] Ghamari S, Höhne C, Becke K et al. S2e-Leitlinie Prävention und Therapie des pädiatrischen Emergence Delir 001/035; aktueller Stand: 11/2016. Im Internet: http://www.awmf.org/ uploads/tx_szleitlinien/001-035I_S2e_paed_Emergence_Delir_2016-11.pdf; Stand: 18.09.2018

[8] Pryzbylo HJ, Pryzbylo JH, Davis AT et al. Acute psychosis after anesthesia: the case for antibiomania. Paediatr Anaesth 2005; 15: 703-705. doi:10.1111/j.1460-9592.2004.01525.x
[9] Saravanarkumar K, Venkatesh P, Bromley P. Delayed onset refractory dystonic movements following propofol anesthesia. Paediatr Anaesth 2005; 15: 597-601. doi:10.1111/j.14609592.2005.01493.x

[10] Quraishi SA, Girdharry TD, Xu SG et al. Prolonged retrograde amnesia following sedation with propofol in a 12-year-old boy. Paediatr Anaesth 2007; 17: 375-379. doi:10.1111/ j.1460-9592.2006.02123.x

[11] Wermelt J, Ellerkmann R. Emergence Delir. Prophylaxe und Therapie. Anästhesiol Intensivmed Notfallmed Schmerzther 2016; 51: 448-457. doi:10.1055/s-0041-106569

[12] Indra S, Haddad H, O'Riordan MA. Short-term Propofol infusion and associated effects on serum lactate in pediatric patients. Pediatr Emerg Care 2017; 33: e118-e121. doi:10.1097/PEC.0000000000001291

[13] Berghmans JM, Poley M, Weber F et al. Does the Child Behavior Checklist predict levels of preoperative anxiety at anesthetic induction and postoperative emergence delirium? A prospective cohort study. Minerva Anestesiol 2015; 81: 145-156

[14] Hino M, Mihara T, Miyazaki S et al. Development and Validation of a Risc Scale for Emergence Agitation after General Anesthesia in Children: A prospective observational study. Anesth Analg 2017; 125: 550-555. doi:10.1213/ ANE.0000000000002126

[15] Short TG, Forrest P, Galletly DC. Paradoxical reactions of benzodiazepines-a genetically determined phenomen? Anaesth Intens Care 1987; 15: 330-345

[16] Dennhardt N, Beck C, Huber D et al. Impact of preoperative fasting times on blood glucose concentration, ketone bodies and acid-base balance in children younger than 36 months: A prospective observational study. Eur J Anaesthesiol 2015; 3: 857-861. doi:10.1097/EJA.0000000000000330

[17] Dennhardt N, Beck C, Huber D et al. Optimized preoperative fasting times decrease ketone body concentration and stabilize mean arterial blood pressure during induction of anesthesia in children younger than 36 months: a prospective observational cohort study. Paediatr Anaesth 2016; 26: 838-843. doi:10.1111/pan.12943

[18] Andersson H, Zarén B, Frykholm P. Low incidence of pulmonary aspiration in children allowed intake of clear fluids until called to the operating suite. Paediatr Anaesth 2015; 25: 770-777. doi:10.1111/pan.12667

[19] Almenrader N, Galante D, Engelhardt T. Correspondence. Emergence agitation: is there a European consensus? $\mathrm{Br}$ J Anaesth 2014; 113: 515-522. doi:10.1093/bja/aeu281

[20] Shin YH, Lee SH, Kim DY. Risperidone in a child wtih untractable emergency delirium: a case report. Korean J Anesthesiol 2016; 69: 623-626. doi:10.4097/kjae.2016.69.6.623

[21] de Grahl C, Luetz A, Gratopp A et al. The paediatric Confusion Assessment Method for the Intensive Care Unit (pCAM-ICU): translation and cognitive debriefing for the German-speaking area. Ger Med Sci 2012; 10: Doc07. doi:10.3205/000158

\section{Bibliografie}

DOI https://doi.org/10.1055/a-0575-0473

Anästhesiol Intensivmed Notfallmed Schmerzther 2018; 53 : 766-776 @ Georg Thieme Verlag KG Stuttgart · New York ISSN 0939-2661 


\section{Punkte sammeln auf CMIE.thieme.de}

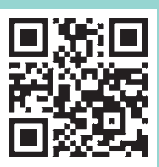

Diese Fortbildungseinheit ist 12 Monate online für die Teilnahme verfügbar.

Sollten Sie Fragen zur Online-Teilnahme haben, finden Sie unter cme.thieme.de/hilfe eine ausführliche Anleitung. Wir wünschen viel Erfolg beim Beantworten der Fragen!

Unter eref/thieme.de/CXAKCHN oder über den QR-Code kommen Sie direkt zum Artikel zur Eingabe der Antworten.

VNR 2760512018154652986

\section{Frage 1}

Präoperative Angst von Kindern kann durch verschiedene Strategien reduziert werden. Welche der im Folgenden genannten Maßnahmen ist dafür geeignet?

A Das Kind erhält keine Informationen vorab zur geplanten Operation und Narkose.

B Das Kind erhält eine medikamentöse Prophylaxe mit Risperidon.

C Das Kind und seine Familie werden intensiv auf den OP-Tag vorbereitet.

D Das Kind erhält eine medikamentöse Prophylaxe mit Flumazenil.

E Das Kind wird blitzartig an der OP-Schleuse von den Eltern getrennt.

\section{Frage 2}

Welchen Vorteil bietet die regelhafte Erfassung des pädiatrischen Emergence Delir (pädED) anhand eines altersadaptierten scores?

A Persönliche individuelle Einschätzungen werden über Zusatzpunkte zum Scorewert addiert.

B Die sichere Unterscheidung zwischen Schmerzen und pädED ist möglich.

C Hunger und Durst als Ursache der postoperativen Unruhe können sicher ausgeschlossen werden.

D Die Inzidenz des pädED wird nachvollziehbar erfasst und kann entsprechend zeitnah behandelt.

E Die Erfassung des Scores am schlafenden Kind ist möglich.

\section{Frage 3}

Welche Aussage ist richtig? Antipsychotika in der Therapie des pädED ...

A können bei langanhaltendem pädED eine Rescue-Strategie darstellen.

B wirken antagonistisch zum Propofol.

$C$ werden niemals verwendet.

D erzwingen einen intensivstationären Aufenthalt.

E dürfen nur durch einen Kinderpsychiater verabreicht werden.

\section{Frage 4}

Das Propofol-Infusionssyndrom (PRIS) ist eine mögliche lebensbedrohliche Komplikation einer Propofol-Anwendung. Welche der folgenden Aussagen in diesem Zusammenhang ist korrekt?

A Eine Anwendung von Propofol über $>48 \mathrm{~h}$ erscheint bei gleichzeitigem NIRS-Monitoring unbedenklich.

B Eine Anwendung von Propofol über $>48 \mathrm{~h}$ erscheint be gleichzeitiger Überwachung der Nierenfunktion unbedenklich.

C Eine Anwendung von Propofol sollte die Höchstmenge von $4 \mathrm{mg} / \mathrm{kgKG} / \mathrm{h}$ bei einer Anwendung > $24 \mathrm{~h}$ nicht überschreiten.

D Genetisches Mitochondrien-Screening vor einer TIVA ist obligat.

E Eltern müssen über die Möglichkeit eines PRIS im Rahmen der Therapie eines pädED aufgeklärt werden.

\section{Frage 5}

Welche altersadaptierte Skala steht zur Detektion eines pädiatrischen Emergence-Delirs (pädED) zur Verfügung?

A PAED-Skala zur Detektion eines pädED

B KUS-Skala zur Detektion eines pädED

C FLACC-Skala zur Detektion eines pädED

D Locatelli-Skala zur gleichzeitigen Detektion von Schmerzen

E visuelle Analogskalen zur Detektion eines pädED durch die Bezugsperson

\section{Frage 6}

In welcher Altersklasse tritt ein pädED am häufigsten auf?
A Säuglinge
B Teenager
C junge Erwachsene
D Schulkinder
E Kleinkinder

\section{- Weitere Fragen auf der folgenden Seite...}




\section{Punkte sammeln auf CME.thieme.de}

Fortsetzung $\ldots$

\section{Frage 7}

Welche der folgenden Substanzen findet in Deutschland keine Anwendung zur medikamentösen Therapie bzw. Prophylaxe eines pädEd?
A Levosimendan
B Propofol
C Midazolam
D Clonidin
E Flumazenil

\section{Frage 8}

Welche der folgenden Aussagen ist korrekt? Geschwisterkinder eines Kindes mit pädED ...

A müssen in die Therapie eines pädED einbezogen werden.

B sollten bei eigenen Narkosen immer eine Risikoprophylaxe erhalten.

C bekommen gleichzeitig nasales Midazolam.

D sollten postoperativ auf ein eventuell verändertes Geschwisterverhalten vorbereitet werden.

E erhalten eine Notfallkarte der DGAI.

\section{Frage 9}

Wodurch ist das Anästhetikum Sevofluran gekennzeichnet?

A Es ist atemwegsreizend.

B Es begünstigt das Auftreten eines pädED.

C Es flutet langsam an und ab.

D Es wird nur selten zur inhalativen Einleitung in der Kinderanästhesie benutzt.

E Es begünstigt ein langsames Aufwachen.

\section{Frage 10}

Welche der folgenden Aussagen ist korrekt? Midazolam ...

A wird hauptsächlich über CYP2D verstoffwechselt.

B hat keine delirogene Wirkung.

$C$ reduziert präoperative Angst.

D darf in Italien niemals zur Therapie eines pädED verwendet werden.

E kann durch Risperidon antagonisiert werden. 